(1)

\title{
纳米颗粒表面活性剂对T型微通道中液滴分裂的影响
}

\author{
齐杰 $^{1 \dagger}$, 彭佳庆 ${ }^{1 \dagger}$, 杨圣贤 ${ }^{2}$, 骆政园 ${ }^{1^{*}}$, 白博峰 ${ }^{1}$
}

1. 西安交通大学动力工程多相流国家重点实验室, 西安 710049;

2. 中国石油化工股份有限公司胜利油田分公司油气开发管理中心, 东营 257001

$\dagger$ 同等贡献

*联系人, E-mail: zy-luo@xjtu.edu.cn

2021-12-15 收稿, 2022-01-30 修回, 2022-02-08 接受, 2022-02-09 网络版发表 国家自然科学基金(51976160)资助

摘要 液滴分裂是微通道中获得单分散小尺寸液滴的重要方法, 纳米颗粒表面活性剂能够包裹液滴, 是制备功能 胶囊的潜在技术手段, 研究纳米颗粒表面活性剂作用下的液滴分裂行为对于获得尺寸更小、分散性更好的功能液 滴具有重要意义. 本文通过微流体可视化实验和理论分析方法, 研究纳米颗粒表面活性剂对液滴分裂的影响规律. 在纳米颗粒表面活性剂作用下, 液滴分裂存在阻塞分裂、过渡态分裂、非阻塞分裂、不分裂 4 种状态; 通过分析液 滴颈部宽度随时间的变化关系, 得出纳米颗粒表面活性剂对液滴分裂的影响机理, 即通过降低界面张力影响挤压 颈缩速率; 通过分析基于液滴尺寸与毛细数的液滴分裂状态分布相图，建立了液滴阻塞分裂与非阻塞分裂的临界 转化理论模型.

关键词液滴分裂, 纳米颗粒, 表面活性剂, 微通道

微流控芯片能够通过微通道内的非混相多相流动 来产生和操纵离散的液滴，控制液滴的大小和生成速 率, 目前被广泛应用于生命科学 ${ }^{[1]}$ 、化学、生物医学 工程 ${ }^{[2]}$ 、石油工程等研究领域 ${ }^{[3]}$. 常规微流控芯片生成 液滴的尺寸一般在几十微米到几毫米之间 ${ }^{[4]}$, 为了进一 步减小液滴尺寸, 得到几微米级别的均质离散液滴, 满 足生化反应、物质封装等要求, 人们常通过设置 $\mathrm{T}$ 型或 Y 型通道结构, 实现已有液滴的分裂 ${ }^{[59]}$. Yang 等人 ${ }^{[10]}$ 通 过设置带有孔喉道的 $\mathrm{T}$ 型微通道结构, 制备了亚微升级 别的液滴(液滴尺寸单分散性较好), 实现了高效、有序 的液滴制备. Link等人 ${ }^{[11]}$ 通过多级 $\mathrm{T}$ 型结构使液滴不断 分裂, 获得了大小均一的微液滴, 数量为原始单通道微 液滴的 $8 \sim 10$ 倍. Bremond等人 ${ }^{[12]}$ 通过设置 $\mathrm{T}$ 型不对称微 通道产生液滴分裂，从而获得具有固定间距的一对液 滴, 实现了液滴对的行为调控.
液滴在微通道内的分裂主要受主通道流动产生的 黏性应力和两相之间毛细力的影响 ${ }^{[13,14]}$. 因此, 微通道 的几何形状、两相流量、液滴初始尺寸、黏度以及界 面张力都是影响液滴分裂的因素. Jullien等人 ${ }^{[15]}$ 通过设 置不同两相流量, 研究了毛细数范围为 $0.0004 \sim 0.02$ 状 态下 T型微通道内液滴的分裂, 指出液滴分裂存在两种 状态, 即阻塞状态与非阻塞状态, 并且指出非阻塞状态 与阻塞状态之间存在临界长度. Chen等人 ${ }^{[16]}$ 研究了 $\mathrm{T}$ 型 微通道内液滴分裂的不同状态，指出液滴分裂主要有 阻塞分裂、非阻塞分裂、不分裂等 3 种状态，提出了液 滴不同状态转换的经验公式. Leshansky等人 ${ }^{[17]}$ 模拟研 究了 $\mathrm{T}$ 型通道内液滴的分裂行为, 利用局部接触角的 Tanner定律，提出液滴颈部宽度变化的理论预测模型. 除此之外, 人们常通过添加表面活性剂降低两液相之 间的界面张力, 使液滴更容易产生变形及分裂 ${ }^{[18]}$. 
除表面活性剂外, 纳米颗粒也被认为是一种良好 的界面改性剂, 可以稳定液滴, 因此它们也可能对液滴 的分裂有重要的影响, 但纳米颗粒在界面处吸附通常 不稳定, 需要通过对其改性提高其吸附能力 ${ }^{[19]}$. 纳米颗 粒表面活性剂(nanoparticle surfactant, NP-surfactant)能 够提升纳米颗粒在界面吸附的稳定性, 它指的是分散 在一个液相中的功能化纳米颗粒和分散在另一个非混 相中的功能化表面活性聚合物可以在流体-流体界面 上自组装, 这比纳米颗粒单独吸附要稳定得多 ${ }^{[20,21]}$. 传 统的分子类表面活性剂具有亲水性和亲脂性基团, 因 此它们可以吸附在流体界面上, 从而降低界面能. 分子 类表面活性剂通常在流体界面上表现出动态组装, 并 在流体界面与本体流体之间表现出频繁的分子交换. 相反, 吸附在流体界面上的纳米颗粒表面活性剂通常 连接在一起, 非常稳定, 大大削弱了流体界面与本体流 体之间的物质交换. 纳米颗粒表面活性剂在各个领域 均具有广阔的应用前景, 例如自组装的纳米颗粒表面 活性剂在界面上可以稳定液滴, 甚至形成覆盖液滴的 粒子单层, 制备胶囊, 因此它们在物质输送应用中具 有重要的潜力. Toor等人 ${ }^{[22]}$ 通过使用流动聚焦型微通 道, 证明了由纳米颗粒表面活性剂稳定的液滴可用于 各种聚合物配体和纳米颗粒的组合, 实现了染料、蛋 白质、纳米颗粒在液滴中的成功封装. 然而, 纳米颗 粒表面活性剂制备的胶囊尺寸一般在几百微米以上, 制备更小尺寸的液滴胶囊需要通过设置分裂段结构, 实现液滴胶囊的进一步分裂，从而获得单分散性更小 的液滴胶囊. 因此, 纳米颗粒表面活性剂对微通道中 液滴分裂的影响是获得更小液滴胶囊必不可少的研究 内容.

本文通过搭建微流控实验系统, 研究了纳米颗粒 表面活性剂对 $\mathrm{T}$ 型微通道中液滴分裂的影响. 通过设置 不同实验组别, 比较了干净油水界面、纳米颗粒、表 面活性聚合物和纳米颗粒表面活性剂对液滴分裂的影 响. 研究发现, 液滴在 $\mathrm{T}$ 型微通道中的分裂存在 4 种不同 的状态, 即阻塞分裂、过渡态分裂、非阻塞分裂及不 分裂. 通过建立相关的理论模型, 得到了纳米颗粒表面 活性剂对液滴分裂行为的作用机理, 为利用液滴分裂 制备更小尺寸的功能液滴提供了理论基础.

\section{1 实验系统与方法}

采用软光刻技术制备了含 $\mathrm{T}$ 型结构的微通道芯片, 利用等离子体法将含通道结构的聚二甲基硅氧烷 (polydimethylsiloxane, PDMS)模具黏接在玻璃片上, 将 硅油通人黏接后的芯片内, 并在 $100^{\circ} \mathrm{C}$ 下加热 $12 \mathrm{~h}$, 保证 通道结构的疏水性, 通过调节连续相及分散相流量比, 在聚焦型微通道内生成液滴后进人 $\mathrm{T}$ 型微通道内完成 分裂. 微通道芯片的几何结构如图1(a)所示, 其中主通 道宽度 $w_{\mathrm{m}}=50 \mu \mathrm{m}$, 分裂微通道宽度 $w_{\mathrm{s}}=30 \mu \mathrm{m}$, 主通道及 分裂微通道过渡处由半径为 $30 \mu \mathrm{m}$ 的圆弧连接, 微通道 高度 $H=60 \mu \mathrm{m}$. 通过相同的高精度注射百(Harvard Apparatus, PhD ultra, USA)将连续相及分散相流体注人微 通道内, 控制不同流量, 实现对液滴尺寸及毛细数的调 控. 使用倒置显微镜(Leica, DMi8A, Germany)及高速摄 影(Photron, Fastcam Mini UX100, Japan)观察并捕捉拍 摄 $\mathrm{T}$ 型通道中的液滴分裂过程, 利用Image $\mathrm{J}$ 软件对液滴 分裂过程中的图像进行处理及分析. 如图1(b)所示, $U$ 代 表来流平均速度, 液滴凹陷区域假设为标准扇形, 其半 径为 $R$, 液滴与分裂通道相交点为边缘位置 $x$, 对应的圆 弧弧度为 $\varphi$, 液滴颈部宽度为 $\delta$, 液滴在分裂通道中的长 度为 $l_{\mathrm{d}}$.

如图1(c)所示, 使用羧基化聚苯乙烯纳米颗粒水溶 液和氨基化混合硅油分别作为分散相和连续相, 混合 溶液浓度为质量百分比, 下文中使用百分数进行表示. 其中, 羧基化聚苯乙烯纳米颗粒(carboxylated polystyrene, COOH-PS, 购于Microspheres Nanospheres)粒径为 $16.5 \mathrm{~nm}$, 利用去离子水 $\left(1.004 \mathrm{cSt}, \rho=0.998 \mathrm{~g} / \mathrm{cm}^{3}\right.$, Millipore Direct-Q)将纳米颗粒水溶液稀释, 浓度分别为 $0.002 \% 、 0.01 \%$. 将 $\alpha, \omega$-二氨基端聚二甲基硅氧烷 $(\alpha$, $\omega$-diamino-terminated polydimethylsiloxane, $\mathrm{NH}_{2}$ PDMS-NH ${ }_{2}$, 购于Sigma-Aldrich, $M_{n}=2500$ )与二甲基硅 油(50 cSt, $\rho=0.983 \mathrm{~g} / \mathrm{cm}^{3}$, 购于Sigma Aldrich)混合, 制 备浓度为 $10 \%$ 和 $20 \%$ 的混合硅油. 之前的研究证 明 $^{[20 ~ 22]}$, 羧酸功能化的纳米颗粒水溶液与氨基功能化 的硅油接触时, 两种功能化试剂会在油-水界面发生酰 胺化反应, 使纳米颗粒稳定吸附在油水界面, 降低界面 张力, Cui等人 ${ }^{[20]}$ 将该物质称为纳米颗粒表面活性剂. 本研究使用界面张力计(JK99C1 Power-each, China) 测量了不同试剂组合下的界面张力系数(表1). 通过设 置4组不同的流体系统: 纯水纯油、仅添加聚合物、 仅添加纳米颗粒、纳米颗粒表面活性剂, 探究了纳米 颗粒表面活性剂对液滴分裂过程的影响. 经测量, 纳 米颗粒及聚合物的存在对流体密度和黏度的影响微 小, 因此在后续理论计算中采用上述纯流体的黏度及 密度. 


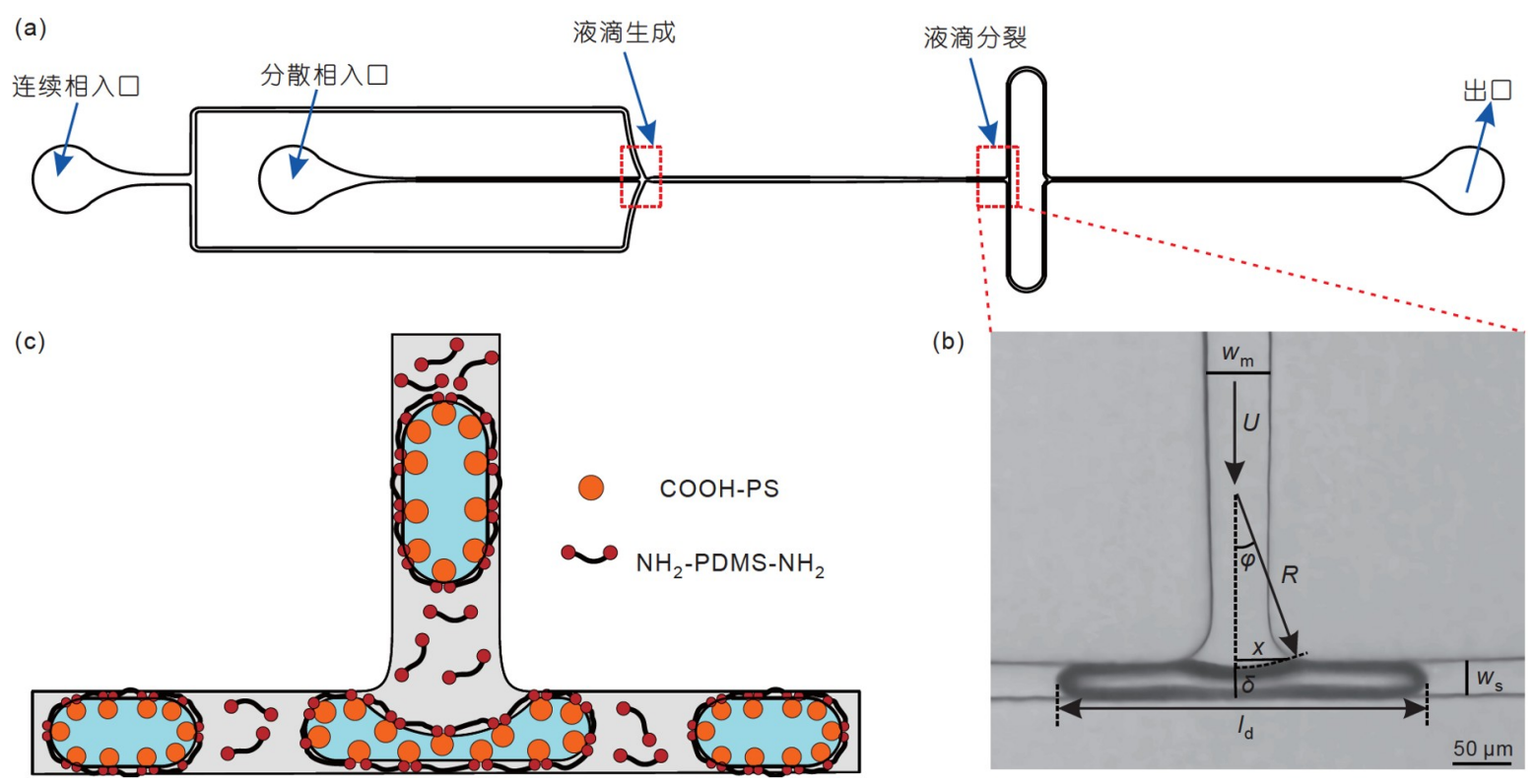

图 1 (网络版彩色)微通道芯片结构及T型微通道内液滴分裂示意图. (a) 微通道结构图; (b) T型微通道结构图; (c) 纳米颗粒表面活性剂包裹于 液滴表面的示意图

Figure 1 (Color online) Schematic diagram of the microchannel chip and droplet splitting in a T-junction microchannel. (a) Overview of the geometry of the microchannel; (b) schematic diagram of a T-junction microchannel structure; (c) schematic diagram of the nanoparticle surfactant distribution on the droplet surface

表 1 不同流体系统中的界面张力系数

Table 1 The interfacial tension for four different fluid systems

\begin{tabular}{cccc}
\hline 流体系统 & $\mathrm{COOH}-\mathrm{PS}(\%)$ & $\mathrm{NH}_{2}-\mathrm{PDMS}^{-\mathrm{NH}_{2}(\%)}$ & $\gamma(\mathrm{mN} / \mathrm{m})$ \\
\hline 纯水纯油 & 0 & 0 & $41.8 \pm 0.6$ \\
仅添加纳米颗粒 & 0.01 & 0 & $42.3 \pm 0.4$ \\
仅添加聚合物 & 0 & 10 & $33.6 \pm 0.6$ \\
& 0.002 & 10 & $31.2 \pm 0.7$ \\
纳米颗粒表面活性剂 & 0.01 & 10 & $25.3 \pm 0.8$ \\
& 0.01 & 20 & $21.2 \pm 0.7$ \\
\hline
\end{tabular}

\section{2 结果与讨论}

\subsection{T型微通道内液滴分裂状态}

图2展示了相同流量下，纯水纯油、仅添加纳米颗 粒、仅添加聚合物、纳米颗粒表面活性剂4种试剂条 件下T型微通道中液滴分裂的时间序列图. 可以看出, 液滴分裂过程可分为 3 个阶段：进人阶段、挤压分裂阶 段和分裂后阶段. 进人阶段从液滴头部进入圆弧段开 始, 在上游流体压力的推动下, 液滴逐渐向下运动进人 $\mathrm{T}$ 型结构并向两边伸展, 直至完全进人 $\mathrm{T}$ 型结构. 当液滴 尾部由凸起圆弧形变直时, 进人挤压分裂阶段, 此阶段 过程中液滴颈部宽度逐步减小，同时液滴的两端头部
沿分裂通道向两侧不断拉伸．当液滴分裂成两个子液 滴后, 进人分裂后阶段, 子液滴尾部由尖雉状逐渐变为 圆弧状, 整个分裂过程结束.

通过对比相同流量、不同试剂作用下的液滴分裂 时间可知, 液滴分裂是一个相对快速的过程，同等流量 下纳米颗粒表面活性剂的生成能够有效减小液滴分裂 整个过程所需时间. 对于进人阶段, 纳米颗粒表面活性 剂的生成能够减小液滴尺寸，相比于其他 3 组试剂，同 等流量下液滴进人阶段所需时间缩短至 $32 \mathrm{~ms}$. 挤压分 裂阶段中，4组试剂条件下所用时间大致相同，纳米颗 粒表面活性剂的生成使挤压分裂阶段时间略微增加. 在分裂后阶段, 不同试剂条件下所用时间差异较小. 


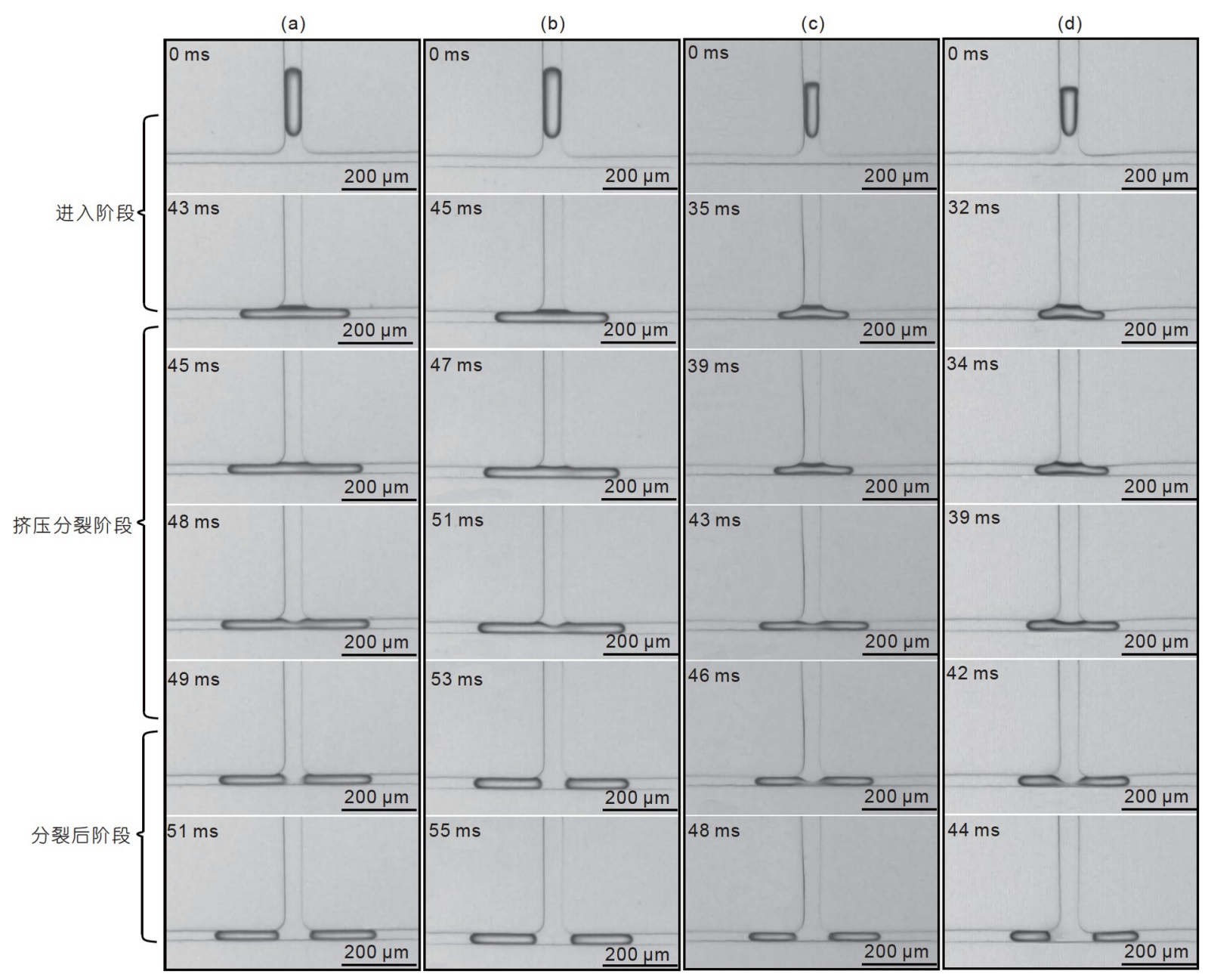

图 2 液滴分裂过程时间序列图. (a) $0 \% \mathrm{NPs}, 0 \% \mathrm{NH}_{2}-\mathrm{PDMS}^{-\mathrm{NH}_{2}}$; (b) $0.01 \% \mathrm{NPs}, 0 \% \mathrm{NH}_{2}-\mathrm{PDMS}_{-} \mathrm{NH}_{2} ;$ (c) $0 \% \mathrm{NPs}_{2}, 10 \% \mathrm{NH}_{2}-\mathrm{PDMS}-\mathrm{NH}_{2}$; (d) $0.01 \% \mathrm{NPs}, 10 \% \mathrm{NH}_{2}-\mathrm{PDMS}-\mathrm{NH}_{2}$. $Q_{\text {water }}=0.3 \mu \mathrm{L} / \mathrm{min}, Q_{\mathrm{oil}}=3 \mu \mathrm{L} / \mathrm{min}$

Figure 2 Time evolution of the droplet splitting process. (a) $0 \% \mathrm{NPs}, 0 \% \mathrm{NH}_{2}-\mathrm{PDMS}-\mathrm{NH}_{2}$; (b) $0.01 \% \mathrm{NPs}, 0 \% \mathrm{NH}_{2}-\mathrm{PDMS}^{-\mathrm{NH}_{2}}$; (c) $0 \% \mathrm{NPs}, 10 \%$ $\mathrm{NH}_{2}$-PDMS-NH ${ }_{2}$; (d) $0.01 \% \mathrm{NPs}, 10 \% \mathrm{NH}_{2}-\mathrm{PDMS}_{-} \mathrm{NH}_{2} \cdot Q_{\text {water }}=0.3 \mu \mathrm{L} / \mathrm{min}, Q_{\text {oil }}=3 \mu \mathrm{L} / \mathrm{min}$

除此之外，实验中观察到了液滴分裂的4种不同状 态(图3), 分别为阻塞分裂、过渡态分裂、非阻塞分 裂、不分裂. 阻塞分裂状态下, 从液滴完全进人分裂通 道直至分裂结束, 液滴始终与通道壁面接触. 过渡态分 裂是指液滴完全进人分裂微通道时，液滴与通道底端 存在弧状间隙，在分裂结束时刻液滴尾端与通道壁面 部分接触. 在非阻塞分裂状态下, 液滴在完全进人分裂 通道到分裂结束, 液滴与通道壁面始终存在间隙. 过渡 态分裂属于阻塞分裂的一种特殊状态，因与通道底端 存在弧状间隙, 受上壁面接触影响, 在毛细力主导的液 滴迅速断裂阶段, 颈部产生双向凹陷程度更大. 不分裂 状态是指液滴在完全进人分裂通道后，直接沿一侧通 道流动, 液滴不发生断裂. 毛细数范围为 $0.02 \sim 0.12$ 时,
在纯水纯油及仅添加纳米颗粒的工况下，液滴分裂均 处于阻塞分裂状态; 在仅添加聚合物及生成纳米颗粒 表面活性剂工况下, 液滴分裂转变为过渡态分裂, 随着 纳米颗粒表面活性剂浓度增加, 液滴分裂状态转变为 非阻塞分裂. 当聚合物浓度为 $20 \%$ 、纳米颗粒浓度为 $0.01 \%$ 、总流量增加至 $7.2 \mu \mathrm{L} / \mathrm{min}$ 以上时，液滴出现不 分裂状态, 继续增大流量后无法生成液滴. 因此, 本文 主要研究阻塞分裂、过渡态分裂、非阻塞分裂三种分 裂状态.

\section{2 液滴分裂颈缩过程的理论分析}

液滴分裂过程中，颈部宽度的变化能够反映挤压 分裂过程中, 不同试剂组别对挤压分裂过程的影响. 以 
挤压分裂段开始时刻作为初始时刻，对液滴颈部宽度 随时间变化作统计分析, 如图4所示. 图4(a)为不同试剂 组别在相同流量下颈部宽度随时间变化的曲线图, 可 以看出，所有试剂条件下颈部宽度随时间变化均存在 两个阶段，即挤压阶段和快速夹断阶段. 挤压阶段内， 颈部宽度随时间呈线性减薄, 减薄速率变化较小, 但当 颈部宽度减薄到一定程度时, 减薄速率迅速加快, 进人 快速夹断阶段. 在快速夹断阶段中, 颈部曲率显著增加, 颈部与端部毛细压差增大, 液滴迅速发生断裂, 完成整 个分裂过程. 此外, 本实验通过调节聚焦型微通道人口 油水流量比, 调控产生液滴的尺寸, 使其进人 $\mathrm{T}$ 型微通 道内完成分裂. 由于不同试剂组别的界面张力不同, 在
相同流量条件下，纯水纯油组别液滴进人分裂通道的 初始长度大于纳米颗粒表面活性剂组别初始长度. 液 滴较长的纯水纯油组别比纳米颗粒表面活性剂组别在 快速夹断阶段的减薄速率更大，所用时间更短. 图 4(b) 为纳米颗粒表面活性剂组别在不同流量比作用下颈部 宽度随时间减薄曲线图，与图4(a)一致，颈部减薄过程 仍然存在挤压及快速夹断两个阶段，但此时随着连续 相与分散相流量比减小, 液滴初始长度增大, 快速夹断 阶段减薄速率反而减小，最终导致颈部分裂时间增加. 产生这一现象的原因可能是在快速夹断阶段，纳米颗 粒聚集在界面收缩最快的颈部，通过产生Marangoni应 力来减缓颈部变薄的速度，阻止界面处液体从颈部流 (a)

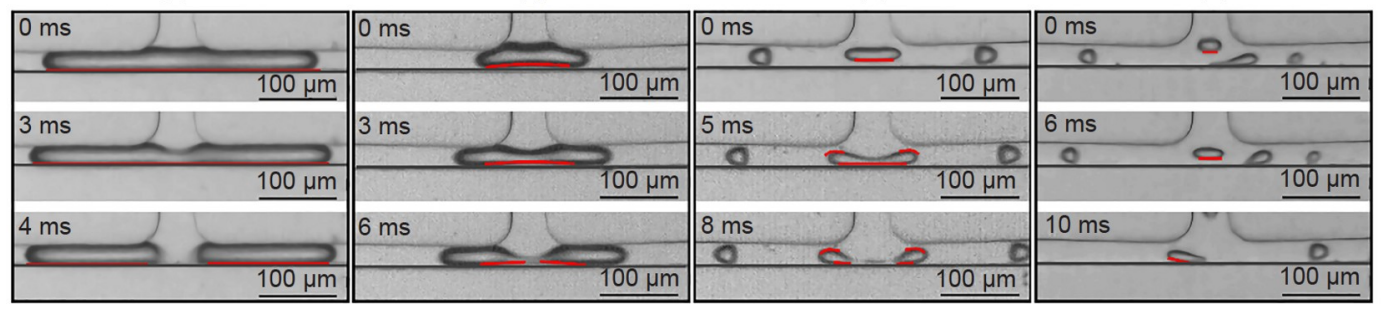

图 3 (网络版彩色) 液滴通过T型微通道时展现的4种不同状态. (a) 阻塞分裂: $0 \% \mathrm{NPs}, 0 \% \mathrm{NH}_{2}-\mathrm{PDMS}-\mathrm{NH}_{2} ; Q_{\text {water }}=0.3 \mu \mathrm{L} / \mathrm{min}, Q_{\mathrm{oil}}=3 \mu \mathrm{L} / \mathrm{min}$. (b) 过渡态分裂: $0 \% \mathrm{NPs}, 10 \% \mathrm{NH}_{2}-\mathrm{PDMS}_{\mathrm{NH}} ; Q_{\text {water }}=0.6 \mu \mathrm{L} / \mathrm{min}, Q_{\text {oil }}=3 \mu \mathrm{L} / \mathrm{min}$. (c) 非阻塞分裂: $0.002 \% \mathrm{NPs}, 10 \% \mathrm{NH}_{2}-\mathrm{PDMS}_{2}-\mathrm{NH}_{2} ; Q_{\text {water }}=$ $1.5 \mu \mathrm{L} / \mathrm{min}, Q_{\text {oil }}=3 \mu \mathrm{L} / \mathrm{min}$. (d) 不分裂: $0.01 \% \mathrm{NPs}, 20 \% \mathrm{NH}_{2}-\mathrm{PDMS}-\mathrm{NH}_{2} ; Q_{\text {water }}=0.15 \mu \mathrm{L} / \mathrm{min}, Q_{\text {oil }}=6 \mu \mathrm{L} / \mathrm{min}$

Figure 3 (Color online) Four different states of the droplet passing through a T-junction microchannel. (a) Blocking splitting: $0 \% \mathrm{NPs}, 0 \% \mathrm{NH}_{2}$ PDMS-NH ${ }_{2} ; Q_{\text {water }}=0.3 \mu \mathrm{L} / \mathrm{min}, Q_{\text {oil }}=3 \mu \mathrm{L} / \mathrm{min}$. (b) Transition state: $0 \% \mathrm{NPs}, 10 \% \mathrm{NH}_{2}-\mathrm{PDMS}_{-\mathrm{NH}_{2}} ; Q_{\text {water }}=0.6 \mu \mathrm{L} / \mathrm{min}, Q_{\mathrm{oil}}=3 \mu \mathrm{L} / \mathrm{min}$. (c) Nonblocking splitting: $0.002 \% \mathrm{NPs}, 10 \% \mathrm{NH}_{2}-\mathrm{PDMS}-\mathrm{NH}_{2} ; Q_{\text {water }}=1.5 \mu \mathrm{L} / \mathrm{min}, Q_{\text {oil }}=3 \mu \mathrm{L} / \mathrm{min}$. (d) Non-splitting: $0.01 \% \mathrm{NPs}_{2} 20 \% \mathrm{NH}_{2}-\mathrm{PDMS}-\mathrm{NH}_{2} ; Q_{\text {water }}$ $=0.15 \mu \mathrm{L} / \mathrm{min}, Q_{\mathrm{oil}}=6 \mu \mathrm{L} / \mathrm{min}$
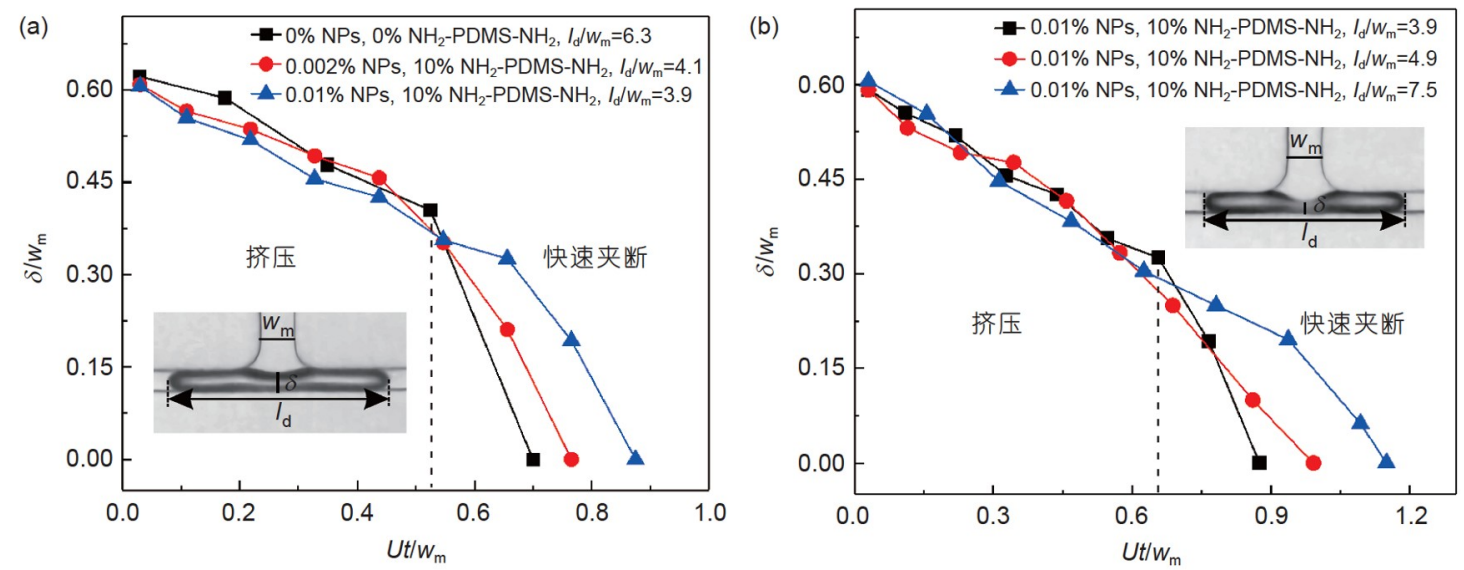

图 4 (网络版彩色)液滴分裂过程中液滴颈部宽度随时间变化曲线图. (a) 不同试剂组别在相同流量条件下颈部减薄曲线图. $Q_{\text {water }}=0.15 \mu \mathrm{L} / \mathrm{min}$, $Q_{\mathrm{oil}}=3 \mu \mathrm{L} / \mathrm{min}$. (b) 纳米颗粒表面活性剂组别在不同流量条件下颈部减薄曲线图. $l_{\mathrm{d}} / w_{\mathrm{m}}=3.9: Q_{\text {water }}=0.15 \mu \mathrm{L} / \mathrm{min}, Q_{\mathrm{oil}}=3 \mu \mathrm{L} / \mathrm{min} ; l_{\mathrm{d}} / w_{\mathrm{m}}=4.9: Q_{\text {water }}=$ $0.3 \mu \mathrm{L} / \mathrm{min}, Q_{\text {oil }}=3 \mu \mathrm{L} / \mathrm{min} ; l_{\mathrm{d}} / w_{\mathrm{m}}=7.5: Q_{\text {water }}=1.5 \mu \mathrm{L} / \mathrm{min}, Q_{\text {oil }}=3 \mu \mathrm{L} / \mathrm{min}$

Figure 4 (Color online) Curves for the neck width of the droplet in droplet splitting. (a) Neck thinning curves of different reagent groups under the same flow condition. $Q_{\text {water }}=0.15 \mu \mathrm{L} / \mathrm{min}, Q_{\text {oil }}=3 \mu \mathrm{L} / \mathrm{min}$. (b) Neck thinning curves of the nanoparticle surfactant group under different flow conditions. $l_{\mathrm{d}} / w_{\mathrm{m}}=3.9: Q_{\text {water }}=0.15 \mu \mathrm{L} / \mathrm{min}, Q_{\text {oil }}=3 \mu \mathrm{L} / \mathrm{min} ; l_{\mathrm{d}} / w_{\mathrm{m}}=4.9: Q_{\text {water }}=0.3 \mu \mathrm{L} / \mathrm{min}, Q_{\text {oil }}=3 \mu \mathrm{L} / \mathrm{min} ; l_{\mathrm{d}} / w_{\mathrm{m}}=7.5: Q_{\text {water }}=1.5 \mu \mathrm{L} / \mathrm{min}, Q_{\text {oil }}=3 \mu \mathrm{L} / \mathrm{min}$ 
出, 并且随着整体流量增加, 减缓颈部变薄的速度趋势 越大，这与Priest等人 ${ }^{[23]}$ 关于分子类表面活性剂的结果 一致.

正如前文所述，连续相及分散相的流量大小会影 响产生的液滴长度及毛细数, 颈部宽度减薄过程中, 液 滴长度及毛细数对液滴颈部宽度减薄速率均有影响. 在液滴分裂过程中，液滴受力包括来流流体挤压力、 液滴端部受到的黏性应力、颈部曲率变化引起的界面 张力、尖端曲率引起的界面张力, 液滴颈部的曲率决 定了液滴临界破裂条件. 图5(a)展示了阻塞分裂状态下 液滴颈部形状变化的时间序列图, 可以看出, 该状态下 液滴颈部以半径为 $R$ 的扇形不断向内凹陷, 进入快速夹 断阶段后, 颈部形状变为漏斗状. 非阻塞分裂状态下, 液滴端部与壁面之间存在较大间隙，液滴表面与壁面 连续相流体速度差异较大, 剪切力导致液滴端部向外 部拉伸. 但本实验对液滴颈部曲率测定显示, 此时颈部 仍以半径为 $R$ 的扇形向内凹陷, 说明剪切力对其造成的 影响相较于颈部曲率变化引起的界面张力影响更小. 由于液滴分裂过程中，不同分裂状态下颈部均以扇形 形状减薄占据较长的时间, 根据Leshansky等人 ${ }^{[17]}$ 建立 的液滴在 $\mathrm{T}$ 型微通道中的分裂几何模型, 假定液滴分裂 过程中颈部以标准扇形不断减薄. 如图1(c)所示, 液滴 在完全进人分裂通道颈缩的过程中, 凹陷区域为标准
扇形, 其半径为 $R$, 液滴与分裂通道相交点为边缘位置 $x$, 对应的圆弧弧度为 $\varphi$, 液滴进人挤压分裂阶段即液滴 颈部与分裂通道平齐时为初始时刻. 在 $t$ 时刻, 凹陷的圆 弧面积为 $S_{\mathrm{d}}=R^{2}(\varphi-1 / 2 \sin (2 \varphi))$, 由质量守恒定律, 凹陷 的液体体积等于 $0 \sim t$ 时刻来流的液体体积, 即 $S_{\mathrm{d}}=U w_{\mathrm{m}} t$, 其中 $U$ 为来流速度, $w_{\mathrm{m}}$ 为主通道宽度. 边缘位置 $x=R \sin \varphi$, 假设在 $x$ 处, 局部接触角的Tanner定律适用 $U^{\prime}=$ $\alpha \gamma \varphi^{3} /(3 \mu)$, 其中 $U^{\prime}$ 为边缘速度, $U^{\prime}=\mathrm{d} x / \mathrm{d} t, \alpha$ 为常数, $\gamma$ 为两 相界面张力, $\mu$ 为连续相黏度. 利用 $w_{\mathrm{m}} / U$ 对时间 $t$ 进行无 量纲处理得到无量纲时间 $\tau, S_{\mathrm{d}}$ 及 $x$ 用 $w_{\mathrm{m}}$ 无量纲处理后得 到 $S_{\mathrm{d}}^{*}$ 和 $x^{*}$ :

$$
\begin{aligned}
& \frac{\mathrm{d} S_{\mathrm{d}}^{*}}{\mathrm{~d} \tau}=\frac{\mathrm{d}}{\mathrm{d} \tau}\left(\frac{2}{3} R^{* 2} \varphi^{3}\right) \approx 1, \\
& \frac{\mathrm{d} x^{*}}{\mathrm{~d} \tau}=\frac{\mathrm{d}\left(R^{*} \varphi\right)}{\mathrm{d} \tau} \approx \frac{\alpha}{3 C a} \varphi^{3},
\end{aligned}
$$

其中, 毛细数 $C a=\mu U / \gamma$. 假设 $R \sim \tau^{a}, \varphi \sim \tau^{b}$, 联立式(1)和(2), 解得

$$
\begin{aligned}
& R^{*} \approx 3^{2 / 7} 7^{3 / 7} / 2^{8 / 7}\left(\frac{\alpha}{3 C a}\right)^{3 / 7} \tau^{5 / 7}, \\
& \varphi \approx 2^{3 / 7} 3^{1 / 7} / 7^{2 / 7}\left(\frac{\alpha}{3 C a}\right)^{-2 / 7} \tau^{-1 / 7} .
\end{aligned}
$$

因主通道及分裂通道连接处为圆弧段，在实际过 (a)

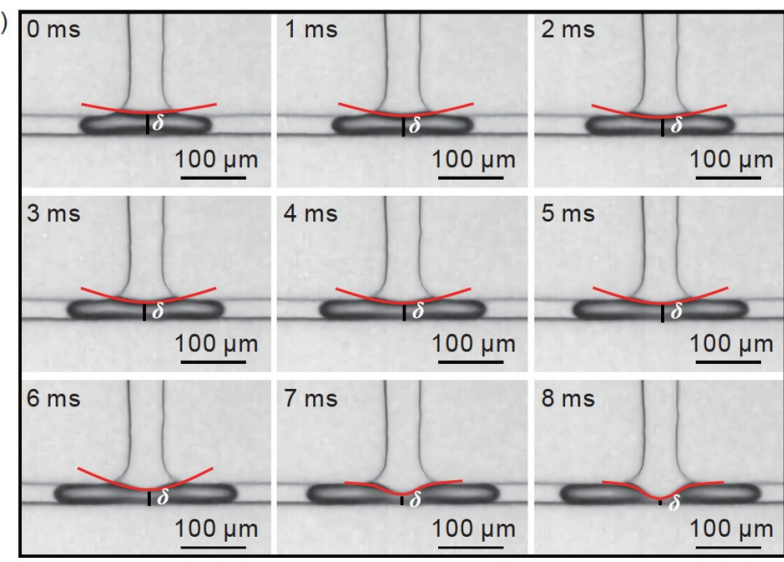

(b)

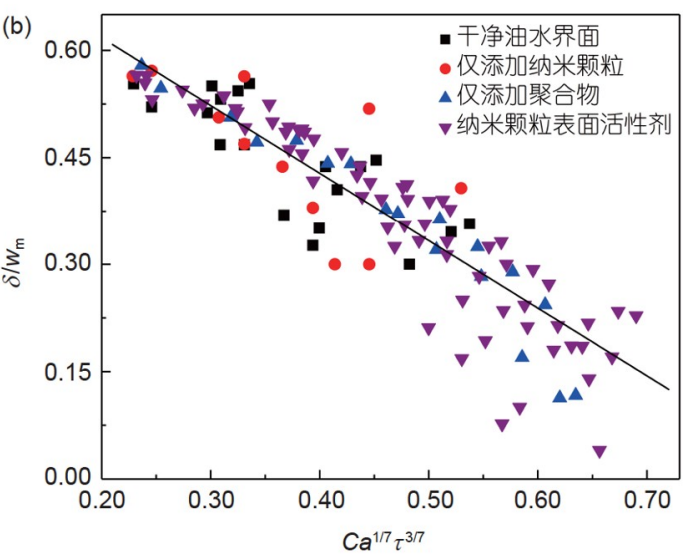

图 5 (网络版彩色)液滴分裂过程中颈部宽度的变化规律. (a) 液滴颈缩过程中颈部形状随时间变化示意图. $0.002 \% \mathrm{NPs}, 10 \% \mathrm{NH}_{2}-\mathrm{PDMS}-\mathrm{NH}_{2}$; $Q_{\text {water }}=1.5 \mu \mathrm{L} / \mathrm{min}, Q_{\mathrm{oil}}=3 \mu \mathrm{L} / \mathrm{min}$. (b) 液滴颈部宽度减薄理论模型拟合结果, 实线为理论拟合, 数据点代表实验结果. 干净油水界面组别: $0 \%$ NPs, $0 \% \mathrm{NH}_{2}-\mathrm{PDMS}-\mathrm{NH}_{2}$. 仅添加纳米颗粒组别: $0.01 \% \mathrm{NPs}, 0 \% \mathrm{NH}_{2}-\mathrm{PDMS}-\mathrm{NH}_{2}$. 仅添加聚合物组别: $0 \% \mathrm{NPs}, 10 \% \mathrm{NH}_{2}-\mathrm{PDMS}-\mathrm{NH}_{2}$. 纳米颗粒表面 活性剂组别: $0.002 \% \mathrm{NPs}, 10 \% \mathrm{NH}_{2}-\mathrm{PDMS}-\mathrm{NH}_{2} ; 0.01 \% \mathrm{NPs}, 10 \% \mathrm{NH}_{2}-\mathrm{PDMS}-\mathrm{NH}_{2} ; 0.01 \% \mathrm{NPs}, 20 \% \mathrm{NH}_{2}-\mathrm{PDMS}_{2}-\mathrm{NH}_{2}$

Figure 5 (Color online) Time evolution of the droplet neck width during the droplet splitting. (a) Schematic diagram of neck shape changing with time during the droplet necking. $0.002 \% \mathrm{NPS}, 10 \% \mathrm{NH}_{2}-\mathrm{PDMS}-\mathrm{NH}_{2} ; Q_{\text {water }}=1.5 \mu \mathrm{L} / \mathrm{min}, Q_{\mathrm{oil}}=3 \mu \mathrm{L} / \mathrm{min}$. (b) Fitting of the theoretical model for the thinning of the droplet neck. The solid line is the theoretical fitting line, and the data points represent experimental results, i.e., pure fluid case: $0 \% \mathrm{NPs}_{0}, 0 \% \mathrm{NH}_{2}-$ PDMS-NH ${ }_{2}$; COOH-PS case: $0.01 \% \mathrm{NPs}, 0 \% \mathrm{NH}_{2}-\mathrm{PDMS}-\mathrm{NH}_{2}$; $\mathrm{NH}_{2}-\mathrm{PDMS}-\mathrm{NH}_{2}$ case: $0 \% \mathrm{NPs}, 10 \% \mathrm{NH}_{2}-\mathrm{PDMS}-\mathrm{NH}_{2}$; NP-surfactant case: $0.002 \%$ NPs with $10 \% \mathrm{NH}_{2}-\mathrm{PDMs}-\mathrm{NH}_{2}, 0.01 \% \mathrm{NPs}$ with $10 \% \mathrm{NH}_{2}-\mathrm{PDMS}-\mathrm{NH}_{2}$ and $0.01 \% \mathrm{NPs}$ with $20 \% \mathrm{NH}_{2}-\mathrm{PDMs}_{2}-\mathrm{NH}_{2}$ 
程中, 当液滴颈部宽度与分裂通道平齐时, 液滴颈部已 经发生了收缩, 如图2所示, 故引人修正系数 $c$ 对颈部宽 度进行修正: $\delta=w_{\mathrm{s}}-R(1-\cos \varphi)+c$. 由于在颈部开始收缩 时刻, 扇形弧度极小, 根据泰勒展开, 此时 $\delta=c_{0}-(1 / 2) R \varphi^{2}$, 其中 $c_{0}$ 为颈缩开始时刻颈部的初始宽度. 根据式(3)和 (4), 可得出颈部宽度及边缘位置随时间及毛细数的变 化关系:

$$
\begin{aligned}
\delta^{*} & =c_{x}^{*}-\frac{1}{2} R^{*} \varphi^{2}=c_{x}^{*}-3^{4 / 7} 7^{1 / 7} / 2^{9 / 7}\left(\frac{\alpha}{3 C a}\right)^{-1 / 7} \tau^{3 / 7} \\
& =C_{\mathrm{A}}-C_{\mathrm{B}} C a^{1 / 7} \tau^{3 / 7} \\
x^{*} & =R^{*} \varphi=3^{3 / 7} 7^{1 / 7} / 2^{5 / 7}\left(\frac{\alpha}{3 C a}\right)^{1 / 7} \tau^{4 / 7}=C_{\mathrm{C}} \mathrm{Ca}^{-1 / 7} \tau^{4 / 7} .
\end{aligned}
$$

使用式(5)对实验数据进行拟合(图5(b)), 得到 $C_{\mathrm{A}}$ 、 $C_{\mathrm{B}} 、 C_{\mathrm{C}}$ 分别为 $0.807 、 0.947 、 0.792$. 从图 5(b)可以看 出, 不同试剂条件下, 各组数据均分布在颈部减薄理论 拟合线附近，表明纳米颗粒表面活性剂主要通过影响 毛细数来影响液滴颈部宽度减薄速率, 与纯水纯油、 仅添加纳米颗粒、仅添加聚合物组别相比, 纳米颗粒 表面活性剂能够显著降低界面张力, 从而增加毛细数, 进一步使液滴分裂颈部减薄速率加快. 在液滴挤压分 裂后期，纳米颗粒表面活性剂及仅添加聚合物作用下 的液滴颈部减薄速率几乎不变, 但纯水纯油组别及仅 添加纳米颗粒情况下, 液滴颈部减薄速率存在骤然加 速过程, 与图4结果一致. 此外, 分裂前期, 数据点与拟 合曲线偏离程度较小, 随着时间不断推进, 数据点偏离 拟合线的程度逐渐变大，这是由于假设中凹陷区域为 扇形圆弧, 但是由图5(a)液滴颈部形状变化可知, 随着 分裂的进行, 凹陷区域逐渐偏离圆弧状, 由扇形转变为 漏斗状, 此时假设中的扇形条件不适用, 产生较大偏差. 与上文一致，当液滴颈部宽度减薄至断裂临界宽度附 近时, 液滴颈部分裂进入快速夹断阶段, 此时液滴颈部 形状迅速变化发生断裂, 产生子液滴.

\section{3 液滴分裂状态的相图分布及转变}

液滴分裂过程是两相黏性力及毛细力的平衡. 由 上述研究可知, 纳米颗粒表面活性剂主要通过影响界 面张力来影响分裂过程, 因此可用毛细数 $\mathrm{Ca}$ 描述流量 变化及不同试剂条件带来的影响. 除此之外, 液滴分裂 状态随液滴尺寸减小逐渐由阻塞分裂向非阻塞分裂转 变, 因此阻塞分裂与非阻塞分裂之间的临界条件可由 液滴尺寸及毛细数进行描述.
主通道与分裂通道之间存在圆弧段，液滴在未完 全进人分裂通道时，边缘位置 $x$ 已与主通道圆弧段壁面 接触, 由于圆弧与主通道之间存在液滴面积为 $S_{\mathrm{c}}$, 得出此 时分裂通道内液滴的初始面积 $S_{0}=\left(l_{\mathrm{d}}-w_{\mathrm{s}}\right) w_{\mathrm{s}}+\pi w_{\mathrm{s}}{ }^{2} / 4-S_{\mathrm{c}}$. 当液滴处于非阻塞分裂与过渡态分裂的临界点时，液 滴颈部宽度减小为 0 , 边缘位置 $x$ 与壁面临界接触, 此时 $S^{\prime}=2 x w_{\mathrm{s}}+\pi w_{\mathrm{s}}{ }^{2} / 4-S_{\mathrm{d}}, S_{\mathrm{d}}$ 为液滴凹陷面积. 由体积守恒定 律可知, $S_{0}=S^{\prime}$, 从而得到 $l_{\mathrm{d}}=2 x-S_{\mathrm{d}} / w_{\mathrm{s}}+w_{\mathrm{s}}+S_{\mathrm{c}} / w_{\mathrm{s}}$. 利用主 通道宽度 $w_{\mathrm{m}}$ 进行无量纲化:

$$
l_{\mathrm{d}}^{*}=2 x^{*}-\frac{5}{3} S_{\mathrm{d}}^{*}+\frac{3}{5}+\frac{5}{3} S_{\mathrm{c}}^{*} .
$$

液滴分裂结束时, 即 $\delta=0$ 时, 根据式(5), 解得 $\tau^{\prime}=$ $0.688 \mathrm{Ca}^{-1 / 3}$. 由式(5)和(6)可知, 此时 $S_{\mathrm{d}}{ }^{*}=\tau^{\prime}=0.688 C \mathrm{Ca}^{-1 / 3}$, $(5 / 3) S_{\mathrm{c}}{ }^{*}=S_{\mathrm{c}} /\left(w_{\mathrm{m}} \times w_{\mathrm{s}}\right)=0.66$, 则阻塞分裂与非阻塞分裂之 间的临界关系式为

$$
l_{\mathrm{d}} / w_{\mathrm{m}}=0.133 \mathrm{Ca}^{-1 / 3}+1.26 \text {. }
$$

图6(a)为液滴分裂不同状态的转化相图, 由上述分 析可知, 过渡态分裂为特殊的阻塞分裂状态. 实线为式 (8)得出的阻塞分裂与非阻塞分裂之间的临界转变理论 线, 由此可知, 该模型能够较好地描述实验中非阻塞分 裂与阻塞分裂之间的转化. 液滴分裂的状态主要取决 于毛细数 $C a$ 和液滴尺寸. 随着液滴尺寸的增加和毛细 数的减小, 液滴会从非阻塞分裂逐渐转变为过渡态分 裂, 最终呈现阻塞分裂状态. 根据实验结果, 阻塞分裂 与过渡态分裂之间存在大致的区间分布，如图6(a)中虚 线所示. 图6(b)展示了不同试剂及流量条件下出现的分 裂状态, 可以看出, 在干净油水界面与仅添加纳米颗粒 工况下, 液滴分裂均为阻塞分裂状态, 这是由于仅添加 纳米颗粒对油水界面张力几乎没有影响. 当仅添加聚 合物时, 液滴分裂向过渡态分裂转变, 这是由于氨基端 聚合物也是一种分子类表面活性剂, 能够降低油水界 面张力, 从而减小液滴尺寸, 增加毛细数. 当同时添加 纳米颗粒与聚合物, 即生成纳米颗粒表面活性剂时, 随 着纳米颗粒表面活性剂浓度的增加, 液滴分裂状态由 阻塞分裂转变为过渡态分裂; 在浓度较高时, 即 $0.01 \%$ $\mathrm{NPs}, 20 \% \mathrm{NH}_{2}-\mathrm{PDMS}-\mathrm{NH}_{2}$ 条件下, 出现非阻塞分裂, 这 是由于纳米颗粒表面活性剂能够显著降低油水界面张 力, 从而减小液滴尺寸, 增大毛细数, 促使相态转变. 综 上所述，纳米颗粒表面活性剂的生成能够使液滴分裂 由阻塞分裂转变为非阻塞分裂, 这一转变主要通过降 低油水界面张力, 减小液滴尺寸来实现. 

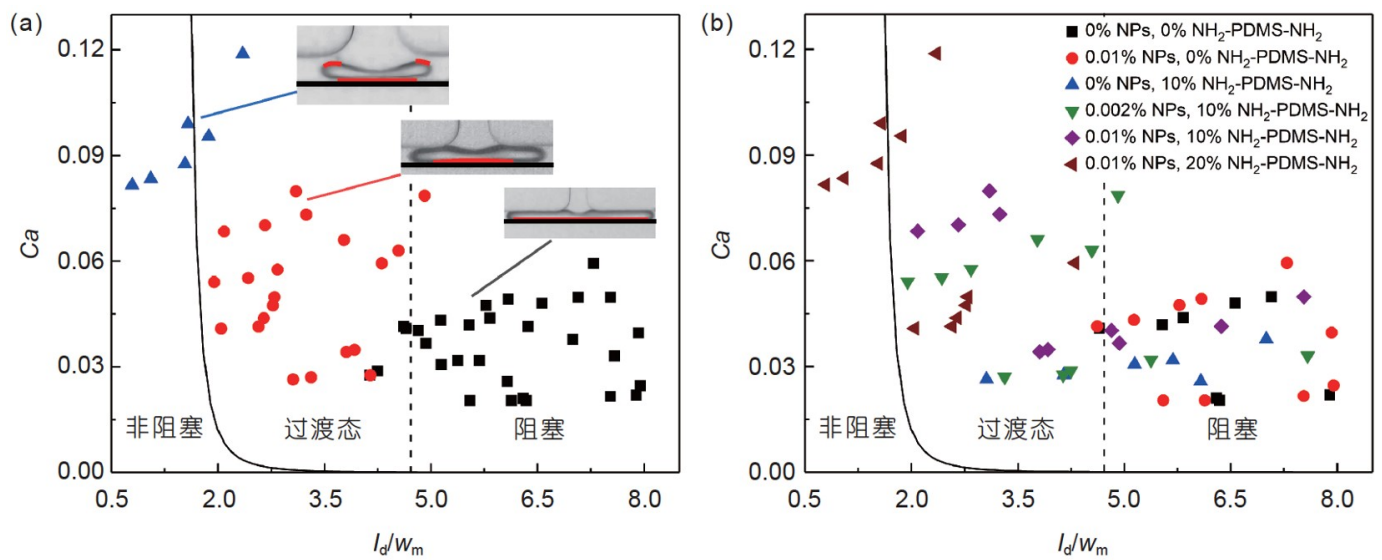

图 6 (网络版彩色)液滴分裂状态的分布相图. (a) 不同液滴分裂状态的分布相图. 实线为阻塞分裂与非阻塞分裂临界转变的理论预测, 虚线为 过渡态分裂与阻塞分裂的分界线. (b) 不同试剂组别下的液滴分裂状态相图. $Q_{\text {water }}=0.075 \sim 1.5 \mu \mathrm{L} / \mathrm{min}, Q_{\mathrm{oil}}=3 \sim 6 \mu \mathrm{L} / \mathrm{min}$

Figure 6 (Color online) Phase diagram for different regimes of droplet splitting. (a) Phase diagram of different droplet splitting states. The solid line is the theoretical prediction for the transition of blocking splitting and non-blocking splitting. The dotted line is the boundary between the transition state and blocking splitting. (b) Phase diagram of droplet splitting states for different reagent groups. $Q_{\text {water }}=0.075-1.5 \mu \mathrm{L} / \mathrm{min}, Q_{\text {oil }}=3-6 \mu \mathrm{L} / \mathrm{min}$

\section{3 结论}

本文搭建了液滴分裂可视化实验平台，研究了纳 米颗粒表面活性剂在 $\mathrm{T}$ 型微通道中的液滴分裂行为, 包 括液滴颈部宽度随时间的变化及液滴分裂过程中的 4 种状态，建立了对液滴分裂状态进行预测的理论模型. 获得的主要结论如下: (1) 液滴分裂存在阻塞分裂、过 渡态分裂、非阻塞分裂、不分裂4种状态，纳米颗粒表 面活性剂的生成能够使液滴分裂由阻塞分裂状态向不
分裂状态依次转变. (2) 建立了液滴分裂过程中颈部宽 度减薄理论模型, 并与实验结果进行拟合, 验证了纳米 颗粒表面活性剂主要通过降低油水界面张力, 增大液 滴分裂挤压前期颈缩速率. (3) 建立了阻塞分裂与非阻 塞分裂之间的临界转变理论模型，与实验结果吻合良 好. 实验证明了纳米颗粒表面活性剂主要通过降低界 面张力, 从而减小液滴尺寸及增大毛细数, 使液滴由阻 塞分裂状态逐步转化为不分裂状态.

\section{参考文献}

1 El-Ali J, Sorger P K, Jensen K F. Cells on chips. Nature, 2006, 442: 403-411

2 Anna S L. Droplets and bubbles in microfluidic devices. Annu Rev Fluid Mech, 2016, 48: 285-309

3 Teh S Y, Lin R, Hung L H, et al. Droplet microfluidics. Lab Chip, 2008, 8: 198-220

4 Shang L, Cheng Y, Zhao Y. Emerging droplet microfluidics. Chem Rev, 2017, 117: 7964-8040

5 Baroud C N, Gallaire F, Dangla R. Dynamics of microfluidic droplets. Lab Chip, 2010, 10: 2032-2045

6 Wang J, Yu D. Asymmetry of flow fields and asymmetric breakup of a droplet. Microfluid Nanofluid, 2015, 18: 709-715

7 Sun X, Zhu C, Fu T, et al. Dynamics of droplet breakup and formation of satellite droplets in a microfluidic T-junction. Chem Eng Sci, 2018, 188: $158-169$

8 Deka D K, Boruah M P, Pati S, et al. Tuning the splitting behavior of droplet in a bifurcating channel through wettability-capillarity interaction Langmuir, 2020, 36: 10471-10489

9 Deng Z L, Li P Y, Zhang X, et al. Semi-obstructed splitting behaviors of droplet in an asymmetric microfluidic T-junction (in Chinese). Acta Phys Sin, 2021, 70: 074701 [队样龙, 李鹏宇, 张璇, 等. T型微通道中液滴半阻塞不对称分裂行为研究. 物理学报, 2021, 70: 074701]

10 Yang Y J, Feng X, Xu N, et al. Generation of sub-femtoliter droplet by T-junction splitting on microfluidic chips. Appl Phys Lett, 2013, 102: 123502

11 Link D R, Anna S L, Weitz D A, et al. Geometrically mediated breakup of drops in microfluidic devices. Phys Rev Lett, 2004, 92 : 054503

12 Bremond N, Thiam A R, Bibette J. Decompressing emulsion droplets favors coalescence. Phys Rev Lett, 2008, 100: 024501

13 Chung C, Lee M, Char K, et al. Droplet dynamics passing through obstructions in confined microchannel flow. Microfluid Nanofluid, 2010, 9: $1151-1163$ 
14 Bai B F, Luo Z Y. Deformation, motion and adhesion of complex droplets under flow (in Chinese). Chin Sci Bull, 2015, 60: 3349-3366 [白博峰, 骆政园. 流场中复杂液滴的变形运动与吸附. 科学通报, 2015, 60: 3349-3366]

15 Jullien M C, Tsang Mui Ching M J, Cohen C, et al. Droplet breakup in microfluidic T-junctions at small capillary numbers. Phys Fluids, 2009, 21: 072001

16 Chen B, Li G, Wang W, et al. 3D numerical simulation of droplet passive breakup in a micro-channel T-junction using the Volume-Of-Fluid method. Appl Therm Eng, 2015, 88: 94-101

17 Leshansky A M, Afkhami S, Jullien M C, et al. Obstructed breakup of slender drops in a microfluidic T junction. Phys Rev Lett, 2012, 108: 264502

18 Riaud A, Zhang H, Wang X, et al. Numerical study of surfactant dynamics during emulsification in a T-Junction microchannel. Langmuir, 2018, 34: $4980-4990$

19 Frijters S, Günther F, Harting J. Effects of nanoparticles and surfactant on droplets in shear flow. Soft Matter, 2012, 8: 6542-6556

20 Cui M, Emrick T, Russell T P. Stabilizing liquid drops in nonequilibrium shapes by the interfacial jamming of nanoparticles. Science, 2013, 342: $460-463$

21 Toor A, Helms B A, Russell T P. Effect of nanoparticle surfactants on the breakup of free-falling water jets during continuous processing of reconfigurable structured liquid droplets. Nano Lett, 2017, 17: 3119-3125

22 Toor A, Lamb S, Helms B A, et al. Reconfigurable microfluidic droplets stabilized by nanoparticle surfactants. ACS Nano, 2018, 12: 2365-2372

23 Priest C, Reid M D, Whitby C P. Formation and stability of nanoparticle-stabilised oil-in-water emulsions in a microfluidic chip. J Colloid Interface Sci, 2011, 363: 301-306 


\title{
Effects of nanoparticle surfactants on droplet splitting in a T-junction microchannel
}

\author{
Jie $\mathrm{Qi}^{1 \dagger}$, Jiaqing Peng ${ }^{1 \dagger}$, Shengxian Yang ${ }^{2}$, Zhengyuan Luo ${ }^{1^{*}} \&$ Bofeng Bai ${ }^{1}$ \\ ${ }^{1}$ State Key Laboratory of Multiphase Flow in Power Engineering, Xi'an Jiaotong University, Xi'an 710049, China; \\ ${ }^{2}$ Shengli Oil Production Plant, Shengli Oilfield Company, SINOPEC, Dongying 257001, China \\ $\dagger$ Equally contributed to this work \\ * Corresponding author, E-mail: zy-luo@xjtu.edu.cn
}

Droplet microfluidics can generate and manipulate discrete droplets through immiscible multiphase flows inside microchannels, which effectively controls the size and generation rate of droplets. Due to its remarkable advantages, droplet microfluidics bears a significant value in an extremely wide range of areas, such as bio(chemical) analysis, particle preparation or petroleum engineering. Droplet splitting is an important form of obtaining monodisperse and small droplets in microfluidic devices, which can obtain smaller droplets to meet the requirements of biochemical reaction and material encapsulation. Nanoparticle surfactants, formed at liquid-liquid interfaces by the interactions between functional groups on nanoparticles and polymers having complementary end-functionality, are recently proposed as an excellent interface stabilizer to cover liquid droplets for applications of substance encapsulation and delivery. Apparently, understanding droplet splitting behavior with consideration of nanoparticle surfactants is of great significance for obtaining smaller and better dispersive functional droplets. In this paper, by using the approach of microfluidic experiment and theoretical analysis, the effects of nanoparticle surfactants on droplet splitting behavior within T-junction microchannels were investigated. Comparisons were conducted among clean droplets and those stabilized by nanoparticles alone, surfaceactive polymers alone and nanoparticle surfactants, respectively. Besides, the effects of concentrations of both nanoparticles and amino-terminal polymers, which determined nanoparticle surfactants concentration on the fluid-fluid interface, on the droplet splitting behavior have been systematically investigated. We have found that the droplet splitting process can be divided into three stages: Entering stage, squeezing stage and post-splitting stage. The generation of nanoparticle surfactants can effectively reduce the time required for droplet splitting. Besides, the nanoparticle surfactants induced reduction of the total split time is mainly attributed to the decrease of the entering time, while no significant change is presented in the squeezing and post-splitting time. The dynamical behavior of droplets splitting under different reagent conditions can be divided into four categories: Blocking splitting, transition state, non-blocking splitting and non-splitting. The blocking splitting state refers to that the droplets are always in contact with the channel wall during the splitting process. Otherwise, there is always a gap between the droplet and the channel wall, which is called non-blocking splitting. Besides, the transition state is a special state of non-blocking splitting, which is in contact with the upper wall of the channel and has a gap with the bottom of the channel. More importantly, by analyzing the transient decreasing of neck width, we found that the thinning rate of the droplet was suddenly accelerated in the process of neck thinning, i.e., the rapid fracture stage. During neck thinning, the depression is fanned out until it reaches a critical width and becomes funnelshaped. By geometric analysis of neck shape, a theoretical model of neck width thinning was obtained, which is applicable to different reagent conditions. Besides, the influence mechanism of nanoparticle surfactants on droplet splitting was obtained, i.e., affecting necking rate via decreasing the interfacial tension. Finally, via analyzing the droplet splitting state, a theoretical formulation was established for predicting the transition of blocking splitting and non-blocking splitting, which shows a good agreement with the experimental data. These findings provide a theoretical basis for the preparation of smaller functional droplets by droplet splitting.

\section{droplet splitting, nanoparticle, surfactant, microchannel}

doi: 10.1360/TB-2021-1308 